\title{
Dermatosis paraneoplásica en relación a la detección temprana del cáncer
}

\author{
Paraneoplastic dermatoses in relation to the early detection of cancer
}

\section{Sr. Editor:}

La dermatosis paraneoplásica es una entidad infrecuente que pertenece al grupo de trastornos de piel asociado a tumores malignos, los cuales se expresan a través de características clínico dermatológicas, únicas según el tipo de neoplasia (1).

La detección de la dermatosis paraneoplásica suele pasar desapercibida pues se manifiestan como problemas leves y poco molestos que no indicarían la necesidad de atención médica; sin embargo, su identificación sería de ayuda para la detección y diagnóstico de una neoplasia oculta, conllevando a un tratamiento oportuno (2).

Presentamos brevemente el caso de un varón de 50 años, natural de Lima, Perú, que acudió a consultorio por una enfermedad de cuatro semanas de inicio insidioso y curso progresivo, caracterizado por la aparición de pequeñas lesiones nodulares violáceas, difusas y pruriginosas, que no cedían a los antihistamínicos, en el tercio distal del miembro inferior izquierdo en la región supra maleolar, con posterior aparición de zonas necróticas. Estas lesiones se extendieron conforme pasaban los días hasta el muslo, comprometiendo el miembro contralateral acompañado de limitación funcional (figura 1).

El paciente fue sometido a un estudio
de inmunofijación, donde se evidenció hipergammaglobulinemia a predominio de $\operatorname{IgG}$ Kappa. Se le realizó una biopsia de piel, donde se halló vasculopatía oclusiva, y un aspirado de medula ósea, donde se evidenció presencia de células de plasmáticas más del $10 \%$ con criterio medular para mieloma múltiple. Posteriormente, el paciente entró en vigilancia con análisis periódicos por consultorio hasta iniciar tratamiento respectivo con quimioterapia.
Las anormalidades vasculares dentro de la clasificación de la dermatosis paraneoplásica son raras pues se estima una asociación entre el 8 al 25\%. Esta patología puede presentar múltiples signos y síntomas como enrojecimiento, púrpura, vasculitis, telangiectasia e isquemia cutánea; los cuales según su agrupación fisiopatológica suelen relacionarse con el tumor subyacente maligno que presenta los pacientes (3).

La vasculitis se presenta con mayor frecuencia en neoplasias hematológicas tipo mieloma múltiple, linfoma, leucemia, histiocitosis maligna y síndromes mielodisplásicos; llegando hasta el $77 \%$ en comparación con los tumores sólidos (4). No obstante, el inicio de las manifestaciones clínicas por dermatosis en relación al tiempo es muy variable, y dependerá del tipo de cáncer, estimándose entre semanas y años.

En el mieloma múltiple la afectación cutánea especifica es rara y se presenta en estadios avanzados asociado a gran masa tumoral, debido a su desarrollo a partir de una lesión osteolítica con extensión directa a

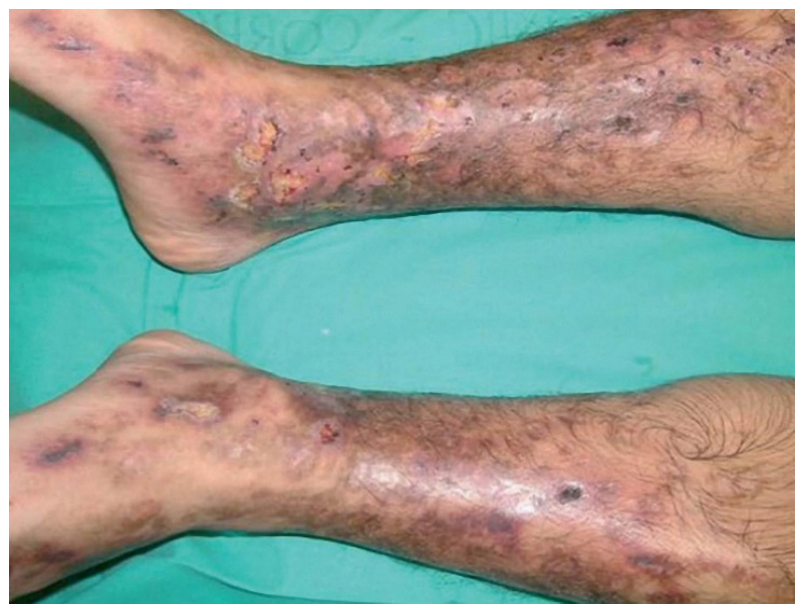

Figura 1. Lesiones nodulares violáceas con áreas de necrosis. 
la piel formando generalmente nódulos o pápulas (5), diferenciándose de la dermatosis neoplásica que se presenta por anticuerpos producidos por la neoplasia hacia las células endoteliales en la pared vascular presentados diversos tipos de lesiones ya descritas.

Los autores creemos que es de gran importancia tener en cuenta las diversas presentaciones de la dermatosis paraneoplásica, primero debido a que el reconocimiento de las mismas podría facilitar la identificación temprana de un cáncer y el tratamiento precoz, aumentando la tasa de supervivencia de los pacientes; y segundo la identificación de la neoplasia y por consiguiente el tratamiento específico mejora el cuadro de la dermatosis paraneoplásica hasta su curación.

\section{Cristian Morán-Mariños ${ }^{1, a}$, Wendy Nieto-Gutierrez ${ }^{2, a}$}

\section{Correspondencia:}

Cristian Andy Morán Mariños

Dirección: Jr. Pira 433 Urbanización el Parque

Naranjal. Lima, Perú.

Correo electrónico: Chriss.L@hotmail.com

Teléfonos: +51931808126

\section{REFERENCIAS BIBLIOGRÁFICAS}

1. Thiers BH, Sahn RE, Callen JP. Cutaneous manifestations of internal malignancy. CA Cancer J Clin. 2009;59(2):73-98. DOI: https://doi. org/10.3322/caac. 20005

2. Martínez AAR, Hernández MAL. Dermatosis paraneoplásicas. Revisión de la bibliografía. Med Int Mex. 2011;27(6):586-95. (Citado el 1 de diciembre del 2018) Disponible en: http://www.medigraphic. $\mathrm{com} / \mathrm{cgi}$-bin/new/resumen.cgi?IDARTICULO= 32622

3. Boyce S, Harper J. Paraneoplastic dermatoses. Dermatologic Clinics. 2002;20(3): 523-32. DOI: https://doi.org/10.1016/S0733-8635(02)00015-3

4. El Tal AK, Tannous Z. Cutaneous vascular disorders associated with internal malignancy.dermatologic Clinics. 2008;26(1): 45-57. DOI: https://doi. org/10.1016/j.det.2007.08.001

5. Requena L. Afectación cutánea específica en pacientes con mieloma múltiple. Estudio clínico-patológico, inmunohistoquímico y citogenético de 40 casos. Actas Dermosifiliogr. 2005; 96(7):424-40. DOI: https://doi.org/10.1016/S0001-7310(05)73107-0

Recibido: 05/12/2018

Escuela profesional de Medicina Humana, Universidad Privada San Juan Bautista, Lima, Perú.

2 Facultad de Medicina Humana, Universidad de San Martin de Porres. Lima, Perú.

a Estudiante de Medicina Humana. 